\title{
Influência do tamanho da leitegada, parição e presença de mumificados na duração da gestação em suínos
}

\author{
Influence of litter size, parity and mummified fetuses on swine gestation length
}

\author{
Ana Paula Gonçalves Mellagi ${ }^{1}$, Mari Lourdes Bernardi ${ }^{2}$, Fernando Pandolfo Bortolozzo ${ }^{1} \&$ Ivo Wentz $^{1}$
}

\begin{abstract}
RESUMO
Como o desencadeamento do parto é dependente do cortisol fetal, o objetivo do estudo foi verificar se o aumento no número de leitões nascidos, observados nos últimos anos, e a ordem de parto influenciam a duração da gestação em suínos. Dados referentes a 21824 partos foram coletados a partir do programa de gerenciamento de dados PigCHAMP ${ }^{\circledR}$. A duração

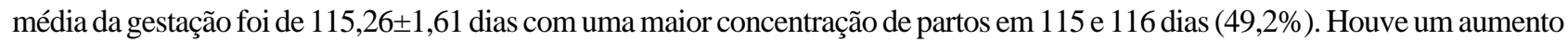
de 0,5 dia na gestação de fêmeas com quatro fetos mumificados $(\mathrm{P}<0,01)$. Observou-se uma correlação significativa $(\mathrm{P}<0,05)$ e negativa $(r=-0,19)$ entre o tamanho da leitegada e a duração da gestação. Fêmeas com maior tamanho de leitegada $(\geq 14$ leitões)

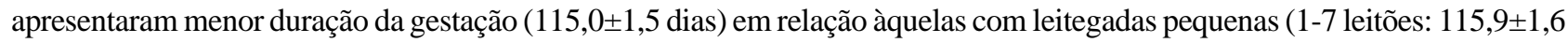
dias). Fêmeas com 11 ou mais leitões concentraram mais de $80 \%$ das suas gestações até 116 dias. Apesar da queda do tamanho da leitegada ser maior no segundo parto, a média do período gestacional foi maior na classe de ordem de parto 6-8. Entretanto, essa diferença tem pouca importância sob o ponto de vista biológico, pois é inferior a meio dia.
\end{abstract}

Descritores: duração da gestação, tamanho da leitegada, parição, mumificados, suínos.

\section{ABSTRACT}

Since the initiation of parturition is dependent of foetal cortisol, the goal of this study was verify if the litter size increase in the last years and the parity influence the pregnancy length in swine. Data concerning 21,824 farrowings were obtained from a backup of the PigCHAMP farm management program. The gestation length mean was $115.26 \pm 1.61$ days, with higher concentration in 115 and 116 days (49.2\%). There was a 0.5 day increased in pregnancy of females with four mummified fetus $(\mathrm{P}<0.01)$. There was a significant $(\mathrm{P}<0.05)$ negative correlation between litter size and duration of pregnancy $(\mathrm{r}=-0.19)$. Females with large litters ( $\geq 14$ piglets) presented lower length pregnancy (115.0 \pm 1.5 days) than females with small litter (1-7 piglets; $115.9 \pm 1.6$ days). Females with 11 or more piglets concentrated $80 \%$ pregnancy length until 116 days. Even the decrease in the litter size is higher in the second parity, the gestation mean was higher in the parity class 6-8. However, this difference have low biology importance, because is below a half day.

Key words: pregnancy length, litter size, parity, mummified, swine. 


\section{INTRODUÇÃO}

Em suínos tem sido relatado que $85 \%$ das gestações duram entre 114-116 dias, com uma amplitude de 110-119 dias [8]. Entretanto, deve-se considerar que o período gestacional pode ser influenciado por alguns fatores como a genética, o tamanho da leitegada e a estação do ano [5,9], além da granja e do ambiente [9].

A manutenção da gestação, em suínos, é dependente da adequada secreção de progesterona pelos corpos lúteos durante todo o período gestacional. $\mathrm{O}$ desencadeamento do parto tem início com a liberação de cortisol a partir dos fetos, o qual estimula a placenta a converter progesterona em estrógeno. Estes níveis elevados de estrógeno estimulam o desenvolvimento de receptores de ocitocina e a secreção de $\mathrm{PGF}_{2} \alpha$ pelo endométrio, causando luteólise. Com a regressão dos corpos lúteos, há uma diminuição nas concentrações de progesterona [5]. A compreensão do processo e dos mecanismos que controlam o parto pode resultar em diminuição de perdas e maior controle da parição, através de auxílio aos leitões. A recente evolução da produção de suínos resultou em um expressivo aumento do número de leitões nascidos totais por fêmea [2]. Este incremento nos nascidos totais também foi seguido de aumento no número de fetos mumificados, que pode estar associado ao prolongamento da gestação [4]. Além disso, alguns autores citam que fêmeas de primeiro parto apresentam gestações mais curtas [9]. Nesse sentido, algumas especulações são feitas sobre uma possível associação dessas alterações no período gestacional.

Desta forma, o objetivo deste trabalho foi verificar se o tamanho da leitegada, a presença de fetos mumificados e a ordem de parto influenciam a duração da gestação em suínos.

\section{MATERIAIS E MÉTODOS}

A avaliação foi baseada em uma unidade produtora de suínos com 5500 fêmeas de uma mesma genética (Agroceres PIC ${ }^{\circledR 1}$ ), localizada na região CentroOeste do Brasil. Foram coletados dados de um período de 2 anos (2002-2003), por backup do programa de gerenciamento de dados PigCHAMP ${ }^{\circledR 2}$. Foi obtido relatório referente à reprodução somente de fêmeas que apresentaram ciclo reprodutivo até o oitavo parto (OP 1 a 8), totalizando 21824 partos avaliados. A expulsão dos fetos antes dos 110 dias de gestação foi considerada como abortamento [8].
Durante o período de análise, a granja não utilizou o método de indução de partos, sendo este aspecto imprescindível para estudar a fisiologia da gestação. A presença de fetos mumificados foi distribuída em 4 classes: 0, 1, 2, 3 e igual e acima de 4 mumificados. O tamanho da leitegada foi dividido em 4 classes: 1 (1-7 leitões), 2 (8-10 leitões), 3 (11-13 leitões) e 4 ( $\geq 14$ leitões). A duração da gestação foi caracterizada pelo intervalo de dias entre a primeira inseminação e o parto, sendo avaliada em 3 classes: curta (110-114 dias), média (115-116 dias) e longa (117120 dias). A frequiência de distribuição das fêmeas de acordo com a duração da gestação e a freqüência de distribuição das classes de duração de gestação de acordo com as classes de tamanho da leitegada, foram obtidas pelo procedimento FREQ do SAS [10]. A comparação dos percentuais de fêmeas com gestação curta, média ou longa, entre as classes de tamanho da leitegada, foi efetuada pelo teste qui-quadrado. A duração média da gestação foi analisada pelo procedimento GLM do SAS e a comparação entre as classes de mumificados, tamanho da leitegada e ordem de parto foram efetuadas pelo teste de Tukey-Kramer.

\section{RESULTADOS}

A distribuição das fêmeas de acordo com a duração da gestação é apresentada na Figura 1. Foi observado que 88,3\% dos partos ocorreram entre 113 e 117 dias de gestação, com a maior concentração entre 115 e 116 dias (49,2\%). A média das gestações foi de $115,26 \pm 1,61$ dias. Houve um percentual de $65,6 \%$ dos partos, no intervalo de 114 a 116 dias de gestação.

A Tabela 1 mostra a distribuição da duração da gestação até e após 115 dias. Observa-se que, com o aumento do número de leitões mumificados, há aumento no percentual de fêmeas com duração da gestação acima de 115 dias. A diferença na média das gestações chega a 12 horas.

Na Tabela 2 é apresentada a distribuição das fêmeas divididas em classes de tamanho da leitegada de acordo com classes de duração da gestação. Houve uma correlação significativa $(\mathrm{P}<0,05)$ negativa $(\mathrm{r}=-0,19)$, entre o tamanho da leitegada e a duração da gestação. Foi observado aumento gradativo no percentual de fêmeas com gestação curta e diminuição simultânea e gradativa no percentual de fêmeas com gestação longa à medida que aumentou o número de leitões na leitegada. Entretanto, do total de fêmeas avaliadas, verificase que $19,9 \%$ são fêmeas com 11 ou mais leitões (classe 


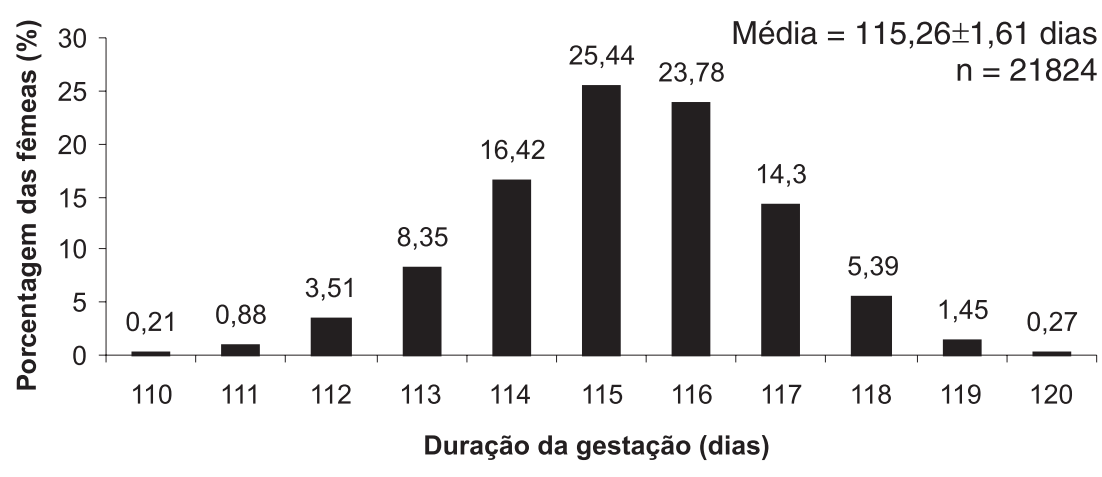

Figura 1. Freqüência de distribuição da duração da gestação de suínos da genética Agroceres PIC ${ }^{\circledR}$, em uma granja localizada no centro-oeste brasileiro no período de 2002 a 2003.

Tabela 1. Duração da gestação e distribuição das mesmas de acordo com a presença de leitões mumificados em suínos da genética Agroceres PIC ${ }^{\circledR}$, em uma granja localizada no Centro-Oeste brasileiro no período de 2002 a 2003.

\begin{tabular}{|c|c|c|c|c|}
\hline \multirow{2}{*}{$\begin{array}{l}\text { Leitões } \\
\text { mumificados }\end{array}$} & \multirow{2}{*}{$\mathbf{n}$} & \multirow{2}{*}{$\begin{array}{l}\text { Duração média } \\
\text { da gestação }\end{array}$} & \multicolumn{2}{|c|}{$\begin{array}{l}\text { Distribuição da duração } \\
\text { da gestação (\%) }\end{array}$} \\
\hline & & & $110-115$ (dias) & $116-120$ (dias) \\
\hline 0 & 16899 & $115,3 \pm 1,6^{a}$ & 55,0 & $45,0^{\mathrm{a}}$ \\
\hline 1 & 3700 & $115,2 \pm 1,6^{a}$ & 55,5 & $44,5^{a}$ \\
\hline 2 & 894 & $115,4 \pm 1,6^{a b}$ & 50,6 & $49,4^{b}$ \\
\hline 3 & 220 & $115,5 \pm 1,6^{a b}$ & 49,5 & $50,4^{\mathrm{ab}}$ \\
\hline$\geq 4$ & 114 & $115,8 \pm 1,6^{b}$ & 43,0 & $57,0^{\circ}$ \\
\hline
\end{tabular}

a,b,c na coluna indicam diferença $(\mathrm{P}<0,01)$.

Tabela 2. Distribuição da duração da gestação de acordo com a classe do tamanho da leitegada, em suínos da genética Agroceres PIC ${ }^{\circledR}$, em uma granja localizada no centro-oeste brasileiro no período de 2002 a 2003.

\begin{tabular}{|c|c|c|c|}
\hline \multirow{2}{*}{$\begin{array}{l}\text { Tamanho da } \\
\text { leitegada }\end{array}$} & \multicolumn{3}{|c|}{ Duração da gestação (dias) } \\
\hline & $\begin{array}{c}\text { Curta }(110-114) \\
n=6409(29,4 \%)\end{array}$ & $\begin{array}{c}\text { Média (115-116) } \\
n=10742(49,2 \%)\end{array}$ & $\begin{array}{l}\text { Longa }(117-120) \\
n=4673(21,4 \%)\end{array}$ \\
\hline $\begin{array}{c}1-7 \text { leitões } \\
\mathrm{n}=2897(13,3 \%)\end{array}$ & $529(18,3 \%)^{a}$ & $1346(46,5 \%)^{\mathrm{a}}$ & $1022(35,2 \%)^{a}$ \\
\hline $\begin{array}{c}\text { 8-10 leitões } \\
\mathrm{n}=5955(27,3 \%)\end{array}$ & $1540(25,9 \%)^{b}$ & $2976(50,0 \%)^{b}$ & $1439(24,1 \%)^{b}$ \\
\hline $\begin{array}{c}11-13 \text { leitões } \\
\mathrm{n}=8250(37,8 \%)\end{array}$ & $2669(32,3 \%)^{c}$ & $4092(49,6 \%)^{b}$ & $1489(18,1 \%)^{c}$ \\
\hline $\begin{array}{c}\geq 14 \text { leitões } \\
n=4722(21,6 \%)\end{array}$ & $1671(35,4 \%)^{d}$ & $2328(49,3 \%)^{b}$ & $723(15,3 \%)^{d}$ \\
\hline
\end{tabular}

3 e 4 juntas) apresentando gestação curta (<115 dias) e 10,1\% apresentaram gestação longa (>116 dias).

A média da duração da gestação de cada classe de leitegada é apresentada na Tabela 3. Houve diferença significativa na duração média da gestação entre todas as classes de tamanho da leitegada, sendo a maior duração observada para as fêmeas com até 7 leitões e a menor duração para as fêmeas com 14 ou mais leitões.

$\mathrm{Na}$ Tabela 4, pode ser observado que, apesar da queda do tamanho da leitegada ser maior no segundo parto, a média do período gestacional foi maior na classe de OP 6-8.

\section{DISCUSSÃO}

O período gestacional foi definido entre 110-120 dias, baseado em estudos prévios que descrevem 99\% das parições no período de 110 a 119 dias de gestação [8]. Portanto, além de serem retiradas da análise, fêmeas com menos de 110 dias de gestação, considerados como abortamento, foram excluídas fêmeas do outro extremo, acima de 120 dias de gestação.

O presente estudo mostra que a medida que aumenta a incidência de fetos mumificados na leitegada, há uma maior duração da gestação. Neste sentido, foi mostrado que há um atraso no desencadeamento do parto quando todos os fetos são decapitados ou hipofisectomizados, mostrando a importância do cortisol fetal [4]. Cabe salientar que o efeito foi mais marcante que o observado no presente estudo, pois as fêmeas com mais de 4 mumificados pariram, em média, somente 0,5 dia após aquelas sem mumificados ou com apenas 1 mumificado. Assim, mesmo com a presença de $>4$ mumificados, os demais leitões vivos geraram estímulo suficiente para desencadear o parto, dentro dos limites considerados fisiológicos. O efeito dos natimor- 
Tabela 3. Duração da gestação de acordo com a classe do tamanho da leitegada, em suínos da genética Agroceres PIC ${ }^{\circledR}$, em uma granja localizada no centro-oeste brasileiro no período de 2002 a 2003.

\begin{tabular}{ccc}
\hline Tamanho da leitegada & $\mathbf{n}$ & Duração da gestação \\
\hline 1-7 leitões & 2897 & $115,9 \pm 1,6^{\mathrm{a}}$ \\
8-10 leitões & 5955 & $115,4 \pm 1,6^{\mathrm{b}}$ \\
11-13 leitões & 8250 & $115,1 \pm 1,5^{\mathrm{c}}$ \\
$\geq$ 14 leitões & 4722 & $115,0 \pm 1,5^{\mathrm{d}}$ \\
\hline a,b,c,d na coluna indicam diferença $(\mathrm{P}<0,0001)$.
\end{tabular}

Tabela 4. Duração da gestação de acordo com a classe de ordem de parto (OP), em suínos da genética Agroceres PIC ${ }^{\circledR}$, em uma granja localizada no centro-oeste brasileiro no período de 2002 a 2003.

\begin{tabular}{cccc}
\hline $\begin{array}{c}\text { Classe de } \\
\text { parição }\end{array}$ & $\mathbf{n}$ & $\begin{array}{c}\text { Tamanho da } \\
\text { leitegada }\end{array}$ & $\begin{array}{c}\text { Duração da } \\
\text { gestação }\end{array}$ \\
\hline OP 1 & 2897 & $11,3 \pm 3,1^{\mathrm{a}}$ & $115,2 \pm 1,6^{\mathrm{ab}}$ \\
OP 2 & 5955 & $10,7 \pm 3,3^{\mathrm{b}}$ & $115,3 \pm 1,6^{\mathrm{a}}$ \\
OP 3-5 & 8250 & $11,6 \pm 3,3^{\mathrm{c}}$ & $115,2 \pm 1,5^{\mathrm{b}}$ \\
OP 6-8 & 4722 & $11,4 \pm 3,3^{\mathrm{a}}$ & $115,6 \pm 1,5^{\mathrm{c}}$ \\
\hline a,b,c na coluna indicam diferença $(\mathrm{P}<0,01)$.
\end{tabular}

tos, por sua vez, não pode ser detalhado, devido à indeterminação do momento da morte, uma vez que o maior percentual de leitões natimortos ocorre durante o parto [1]. Assim, para análise subseqüente do efeito do tamanho da leitegada, foram considerados os leitões nascidos vivos e os natimortos, sendo os fetos mumificados desconsiderados.

O percentual encontrado de $65,6 \%$ dos partos, no intervalo de 114 a 116 dias de gestação, foi inferior à concentração de $85 \%$ relatada na literatura [8], nesse mesmo intervalo. Porém, quando considerase o período normal da gestação de uma granja, avalia-se a duração média com o intervalo de 2 a 3 dias antes e depois. Neste sentido, considerando o intervalo médio e o desvio padrão obtidos na presente avaliação de $115 \pm 2$ dias, observa-se uma concentração de $88,49 \%$ das gestações no período. A baixa correlação encontrada, entre o tamanho da leitegada e a duração da gestação, corrobora com dados anteriores de que o tamanho da leitegada teve baixa influência na duração da gestação [3].
Nos trabalhos que avaliaram a duração da gestação, normalmente é considerado o dia da primeira inseminação/cobertura como dia zero $[7,8]$. No entanto, cabe ressaltar que, em nenhum desses trabalhos foi levado em conta o intervalo entre o momento da ovulação e o parto. Como se sabe, o intervalo entre o início do estro e o momento da ovulação varia de 10 a 58 horas [11], chegando, em alguns casos, até 88 horas [12]. Portanto, essa amplitude individual relacionando o início do estro e a ovulação pode ser de até 3 dias. Estes aspectos deveriam ser considerados quando se avalia a duração da gestação, compreendendo o período entre o início do estro ou primeira cobertura e o parto. Além disso, ao comparar duração média de gestação entre granjas, é importante definir o "dia zero". Ou seja, saber se é o dia do início do estro, ou o dia da primeira cobertura. Para isto, é fundamental conhecer o protocolo de cobertura nas diferentes ordens de parto e, se existem estratégias diferenciadas de inseminação de acordo com o intervalo desmame-estro. Na maioria dos protocolos, as leitoas são inseminadas no início do estro e porcas no turno seguinte. Isto porque a ovulação da leitoa é mais precoce em relação ao início do estro [6]. O protocolo de inseminações de acordo com o intervalo desmame-estro (IDE) deve ser abordado, uma vez que a duração do estro e o intervalo do início do estro à ovulação diminuem com o aumento do IDE [12]. Todos estes fatores podem mascarar as médias das durações da gestação, uma vez que a definição do "dia zero" é diferente, mas o dia do parto (expulsão dos fetos) é o mesmo entre as granjas. Com isso, a duração média da gestação entre as granjas, ou até mesmo entre as fêmeas da mesma propriedade, pode ser influenciada.

De acordo com os dados obtidos, a intensificação no manejo de assistência ao parto, nas gestações com até 116 dias, implicaria em maior cuidado em mais de $80 \%$ das fêmeas com 11 ou mais leitões, que representam $49 \%$ das fêmeas avaliadas e $59 \%$ dos leitões nascidos. Cabe ressaltar que fêmeas com leitegadas grandes apresentam maior risco de natimortalidade, que poderia, assim, ser reduzida com melhor assistência ao parto [1]. Entretanto, cerca de $10 \%$ das fêmeas avaliadas foram aquelas com leitegadas grandes (classes 3 e 4) e com parto atrasado. Isto pode comprometer a taxa de natimortos intra-parto, pois há partos com muitos leitões fora do período esperado. 
Foi verificado que, próximo ao parto, a concentração de estrógeno, necessário para secreção de $\mathrm{PGF}_{2} \alpha$ [5], é maior nas fêmeas com leitegadas maiores comparando às fêmeas com poucos leitões [7]. Os dados apresentados na Tabela 3 poderiam sugerir que um maior número de leitões seria mais eficaz para o início das alterações hormonais que induzem o parto. Entretanto, sob condições práticas, essa diferença entre categorias com menos de 8 leitões ou acima de 14 leitões é inferior a um dia.

A média do período gestacional de acordo com a ordem de parto foi menor para as classes de OP $1 \mathrm{e}$ 3-5, diferindo do exposto anteriormente, em que as primíparas possuem gestações mais curtas [9]. No presente estudo, mesmo com a diminuição do tamanho da leitegada na segunda parição, denominada de síndrome do segundo parto, a gestação mais longa foi observada nas fêmeas entre 6-8 partos. Porém, a dife- rença significativa encontrada de 0,4 dia tem pouca importância biológica.

\section{CONCLUSÕES}

Fêmeas com maior tamanho de leitegada apresentaram menor duração da gestação (considerando como dia zero o dia da $1^{\text {a }}$ inseminação) em relação àquelas com leitegadas pequenas, mas essa diferença não ultrapassa um dia. Fêmeas com 11 ou mais leitões concentram mais de $80 \%$ da duração de gestação até 116 dias. A presença de mais de 4 fetos mumificados pode aumentar a duração da gestação. Apesar da queda do tamanho da leitegada ser maior no segundo parto, a média do período gestacional foi maior nas parições 6 a 8 .

\section{NOTAS INFORMATIVAS}

${ }^{1}$ Agroceres PIC ${ }^{\circledR}$, Patos de Minas, Brasil.

${ }^{2}$ PigCHAMP ${ }^{\circledR}$, Minnesota, Estados Unidos.

\section{REFERÊNCIAS}

1 Borges V., Bernardi M.L., Bortolozzo F.P. \& Wentz I. 2005. Risk factors for stillbirth and foetal mummification in four Brazilian swine herds. Preventive Veterinary Medicine. 70: 165-176.

2 Bortolozzo F.P., Wentz I., Kummer R., Amaral Filha W.S. \& Bernardi M.L. 2005. Como melhorar a eficiência reprodutiva de matrizes suínas em granjas com alta performance. In: Anais do $16^{\circ}$ Congresso Brasileiro de Reprodução Animal (Goiânia, GO). pp.1-14.

3 Day B.N. 1980. Parturition. In: Morrow D.A. (Ed). Current Therapy in Theriogenology: diagnosis, treatment and prevention of reproductive diseases in animals. Philadelphia: Saunders Company, pp.1064.

4 Dziuk F. 1959. Reproduction in the pig. In: Cupps, P. T. (Ed). Reproduction in domestic animals. 4th ed. San Diego: Academic Press, pp.471-489.

5 First N.L., Lohse J.K. \& Nara B.S. 1982. The Endocrine Control of Parturition. In: Control of Pig Reproduction. London: Butterworths, pp.311-342.

6 Kummer R., Amaral Filha W.S., Wentz I. \& Bortolozzo F.P. 2006. Aspectos a serem observados no momento da cobertura da leitoa. In: Bortolozzo F.P. \& Wentz I. (Eds). Suinocultura em ação 3 - A fêmea suína de reposição. Porto Alegre: Pallotti, pp.117-127.

7 Martin P.A., Bevier G.W. \& Dziuk P.J. 1977. The effect of number of corpora lutea on the length of gestation in pigs. Biology of Reproduction. 16: 712-717.

8 Meredith M.J. 1995. Pig Breeding and Infertility. In: Meredith M.J. (Ed). Animal Breeding and Infertility. Bodmin: Blackwell Science, pp.278-353.

9 Muirhead M.R. \& Alexander T.J.L. 2001. Manejo y tratamiento de la enfermedad en el período del parto y lactación. In: Muirhead M.R. \& Alexander T.J.L. (Eds). Manejo sanitario y tratamiento de las enfermedades del cerdo. Buenos Aires: Inter.Medica, pp.263-322.

10 SAS Institute INC. 2000. SAS User's Guide: Statistical Analysis System, Release 8.0, Cary, North Carolina, U.S.A.

11 Soede N.M., Wetzels C.C. H., Zondag W., De Koning M.A.I. \& Kemp B. 1995. Effects of time of insemination relative to ovulation, as determined by ultrasonography, on fertilization rate and accessory sperm count in sows. Journal of Reproduction and Fertility. 104: 99-106.

12 Weitze K.F., Wagner-Reitschel H., Waberski D., Richter L. \& Krieter J. 1994. The onset of heat after weaning, heat duration, and ovulation as major factors in AI timing in sows. Reproduction of Domestic Animals. 29: 349-363.

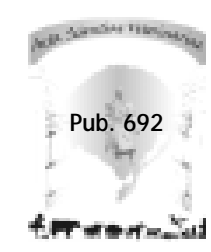

